\title{
Comparative Examination of CO-OFDM Formats for Varied Fiber-Length and Bit Rate
}

\author{
Vikas Kaushik ${ }^{1}$, Himanshi Saini ${ }^{2}$ \\ ${ }^{1}$ Research Scholar, ${ }^{2}$ Assistant Professor, ECE Department, DCRUST, Murthal, India \\ 11dcrust.vikas@gmail.com, ${ }^{2}$ himanshi.ece@dcrustm.org
}

\begin{abstract}
Use of multiple carriers in Orthogonal Frequency Division Modulation (OFDM) makes it bandwidth efficient and suitable for modern communication such as Long-Term Evolution (LTE), Digital Subscriber Line (DSL) internet access, optical fiber communication, digital television, audio broadcasting etc. In this paper, four modulation formats by using Coherent Optical Orthogonal Frequency Division Modulations (CO-OFDM) have been analyzed. These are Single Port Dual Polarization (SP DP) 16 QAM, Single Port Single Polarization (SP SP) 16 QAM, Dual Port Single Polarization (DP SP) QPSK 16-QAM and Single Port Dual Polarization (SP DP) QPSK. Operation characteristics such as Bit Error Rate (BER) versus fiber-length and BER versus bit rate have been examined. The range of fiber length used for this investigation is from $0 \mathrm{Km}$ to $1000 \mathrm{Km}$ with loop count equal to 2 and the range of bit rate of operation is from $20 \mathrm{Gbps}$ to $200 \mathrm{Gbps}$. The present investigation helps in determining the relative suitability of CO-OFDM formats at various bitrates of operation for different fiber lengths.
\end{abstract}

Keywords: CO-OFDM, BER, Single Polarization, Multicarrier Modulation, QAM, QPSK

\section{Introduction}

With increase in the new video-centric applications, there is a need to update information infrastructure because these new applications lead to increase the internet traffic at a rate of $75 \%$ per year. Digital modulation techniques can be single carrier or multicarrier. In multicarrier technique, the data are carried through many closely spaced subcarriers [1]. OFDM is the special class of MCM (Multi-Carrier Modulation) system. MCM system gained popularity for optical communication in longhaul transmission for both CD (Coherent Detection) and DD (Direct Detection) [1][2].

Various research groups have performed experiments on CO-OFDM (Coherent Optical OFDM) and have declared the optical OFDM as the next generation transmission system especially of $100 \mathrm{~Gb} / \mathrm{s}$ Ethernet transport [3]. If OFDM signals are compared with other multi-carrier multiplexing technologies, then it proves to be better in terms of spectral efficiency and anti-multipath interference ability because OFDM signals follow orthogonality in both time and frequency domains [4]. Another specialty of OFDM is that by using it the complexity of transmitters and receivers can be transferred from the analog to the digital domain [5]. Efficient spectral control of the transmitter's output and very less out-of-band energy offered by OFDM could increase spectral efficiency by $50 \%$ in comparison to the WDM systems having 32-GHz channels on a 50-GHz grid [6]. The suitability of CO-OFDM for long haul networks lies in the fact that it shows improved dispersion tolerance and superiority in OSNR requirements [7]. It is assumed that the advantage of the OFDM are due to the following two properties. The first one property is scalable spectrum partitioning. There is scalability of spectrum of subcarriers, of sub-band and of the whole transmission system. This scalability provide flexibility in designing the devices, sub-systems and systems when compare it to single-carrier transmission. The adaptation of pilot subcarriers is the second property of OFDM. The rapid and convenient ways for channel and phase estimation are enabled by this property with the data carriers [8]. Optical OFDM has similarities as well as differences with the RF counterpart. However, the two problems, namely sensitivity to phase/frequency noise and high peak-to-average power ratio (PAPR) are there with the OFDM transmission. On the other hand, the optical channel has also some problems like non linearity and dispersion which are not present in RF system [9][10]. The four formats of CO-OFDM chosen here for investigation are: (1) Single Port Dual Polarization (SP DP) 16 QAM, (2) Single Port Single Polarization (SP SP) 16 QAM, (3) Dual Port Single Polarization (DP SP) QPSK 16-QAM and (4) Single Port Dual Polarization (SP DP) QPSK. All these OFDM formats use coherent detection method at receiver.

This article has been organized in four sections. These are introduction, CO-OFDM experimental setup, results and conclusion. The next section is about the experimental setup having circuit diagram and related signals. The third section explains outcomes of simulation of these circuits and last section concludes the article.

\section{Experimental Setup}

The circuit diagrams implemented in OptiSystem version 14 are shown in figures 1, 2, 3 and 4. The four formats of COOFDM chosen here for investigation are: (1) Single Port Dual Polarization (SP DP) 16 QAM, (2) Single Port Single Polarization (SP SP) 16 QAM, (3) Dual Port Single Polarization (DP SP) QPSK 16-QAM and (4) Single Port Dual Polarization (SP DP) QPSK. All these OFDM formats use coherent detection method at receiver. 
The simulation parameters set in the software for different CO-OFDM formats are shown in table 1. The main simulation parameters are layout parameters, loop control and fiber parameters. The loop control and fiber parameters are common to all modulation formats. The set of values for bit-rate of operation and fiber length used for simulation are 20, 40, 60, 80, 100, 200 and 0, 100, 300, 500, 1000 respectively and units are Gbps and $\mathrm{Km}$ respectively. The loop count for fiber is set equal to 2 .

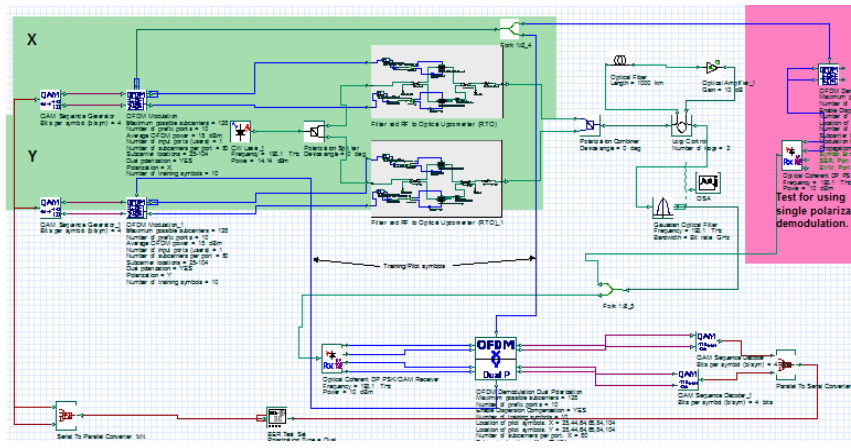

Fig.1. Schematic of SP DP 16 QAM CO-OFDM

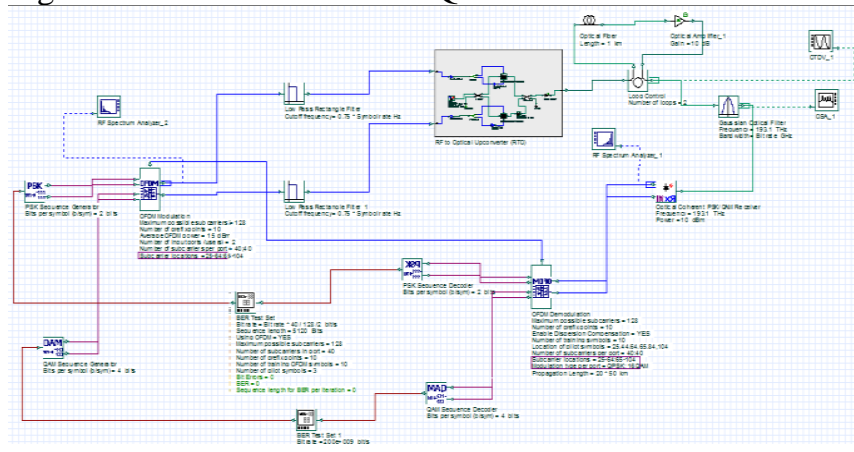

Fig.3. Schematic of DP SP QPSK 16 QAM CO-OFDM

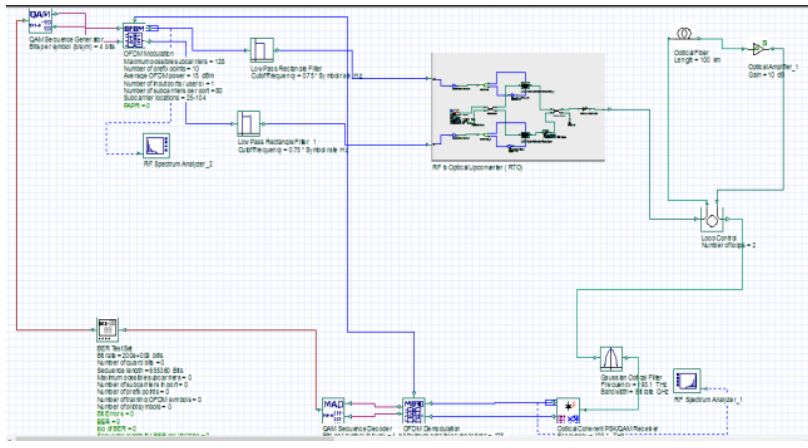

Fig.2. Schematic of SP SP 16 QAM CO-OFDM

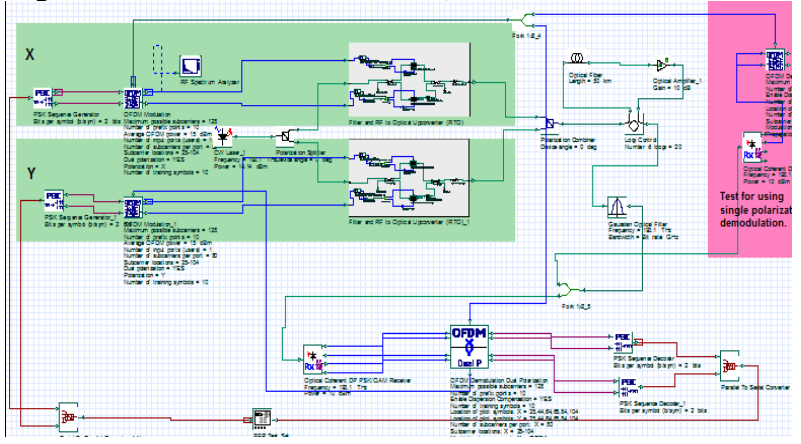

Fig.4. Schematic of SP DP QPSK CO-OFDM

\subsection{Simulation Parameters for CO-OFDM Formats:}

Some layout parameters are needed to be fixed by assigning proper value in order to create a suitable simulation environment for obtaining precise characteristics. Some parameters are inter-related i.e. if one parameter changes then other related parameters will have also to be changed. Table 1 shows values of various parameters for different COOFDM formats.

Table 1: Simulation Parameters employed for four CO-OFDM Formats

\begin{tabular}{|c|c|c|c|c|c|c|}
\hline \multicolumn{7}{|c|}{ Layout Parameters SP DP 16 QAM CO-OFDM } \\
\hline Bit Rate & $20 \mathrm{Gbps}$ & $40 \mathrm{Gbps}$ & $60 \mathrm{Gbps}$ & $80 \mathrm{Gbps}$ & $100 \mathrm{Gbps}$ & $200 \mathrm{Gbps}$ \\
\hline Sequence Length & 32768 & 32768 & 32768 & 32768 & 32768 & 32768 \\
\hline Samples per bit & 1 & 1 & 1 & 1 & 1 & 1 \\
\hline Number of Samples & 32768 & 32768 & 32768 & 32768 & 32768 & 32768 \\
\hline Sample Rate & $20 \mathrm{Gbps}$ & $40 \mathrm{Gbps}$ & $60 \mathrm{Gbps}$ & $80 \mathrm{Gbps}$ & $100 \mathrm{Gbps}$ & $200 \mathrm{Gbps}$ \\
\hline Symbol rate & $2.5 \mathrm{Gbps}$ & $5 \mathrm{Gbps}$ & $7.5 \mathrm{Gbps}$ & $10 \mathrm{Gbps}$ & $12.5 \mathrm{Gbps}$ & $25 \mathrm{Gbps}$ \\
\hline Sensitivity & $-100 \mathrm{~dB}$ & $-100 \mathrm{~dB}$ & $-100 \mathrm{~dB}$ & $-100 \mathrm{~dB}$ & $-100 \mathrm{~dB}$ & $-100 \mathrm{~dB}$ \\
\hline Resolution & 0.1 & 0.1 & 0.1 & 0.1 & 0.1 & 0.1 \\
\hline \multicolumn{7}{|c|}{ Layout Parameters SP SP 16 QAM CO-OFDM } \\
\hline Bit Rate & $20 \mathrm{Gbps}$ & $40 \mathrm{Gbps}$ & $60 \mathrm{Gbps}$ & $80 \mathrm{Gbps}$ & $100 \mathrm{Gbps}$ & $200 \mathrm{Gbps}$ \\
\hline Sequence Length & 32768 & 32768 & 32768 & 32768 & 32768 & 32768 \\
\hline Samples per bit & 1 & 1 & 1 & 1 & 1 & 1 \\
\hline Number of Samples & 32768 & 32768 & 32768 & 32768 & 32768 & 32768 \\
\hline Sample Rate & $20 \mathrm{Gbps}$ & $40 \mathrm{Gbps}$ & $60 \mathrm{Gbps}$ & $80 \mathrm{Gbps}$ & $100 \mathrm{Gbps}$ & $200 \mathrm{Gbps}$ \\
\hline Symbol rate & $5 \mathrm{Gbps}$ & $10 \mathrm{Gbps}$ & $15 \mathrm{Gbps}$ & $20 \mathrm{Gbps}$ & $25 \mathrm{Gbps}$ & $50 \mathrm{Gbps}$ \\
\hline Sensitivity & $-100 \mathrm{~dB}$ & $-100 \mathrm{~dB}$ & $-100 \mathrm{~dB}$ & $-100 \mathrm{~dB}$ & $-100 \mathrm{~dB}$ & $-100 \mathrm{~dB}$ \\
\hline Resolution & 0.1 & 0.1 & 0.1 & 0.1 & 0.1 & 0.1 \\
\hline
\end{tabular}




\begin{tabular}{|c|c|c|c|c|c|c|}
\hline Bit Rate & $20 \mathrm{Gbps}$ & $40 \mathrm{Gbps}$ & $60 \mathrm{Gbps}$ & $80 \mathrm{Gbps}$ & $100 \mathrm{Gbps}$ & $200 \mathrm{Gbps}$ \\
\hline Sequence Length & 32768 & 32768 & 32768 & 32768 & 32768 & 32768 \\
\hline Samples per bit & 1 & 1 & 1 & 1 & 1 & 1 \\
\hline Number of Samples & 32768 & 32768 & 32768 & 32768 & 32768 & 32768 \\
\hline Sample Rate & $20 \mathrm{Gbps}$ & $40 \mathrm{Gbps}$ & $60 \mathrm{Gbps}$ & $80 \mathrm{Gbps}$ & $100 \mathrm{Gbps}$ & $200 \mathrm{Gbps}$ \\
\hline Symbol rate & 10 Gbps & $10 \mathrm{Gbps}$ & $15 \mathrm{Gbps}$ & $20 \mathrm{Gbps}$ & $25 \mathrm{Gbps}$ & 50 Gbps \\
\hline Sensitivity & $-100 \mathrm{~dB}$ & $-100 \mathrm{~dB}$ & $-100 \mathrm{~dB}$ & $-100 \mathrm{~dB}$ & $-100 \mathrm{~dB}$ & $-100 \mathrm{~dB}$ \\
\hline Resolution & 0.1 & 0.1 & 0.1 & 0.1 & 0.1 & 0.1 \\
\hline \multicolumn{7}{|c|}{ Layout Parameters SP DP QPSK CO-OFDM } \\
\hline Bit Rate & $20 \mathrm{Gbps}$ & $40 \mathrm{Gbps}$ & $60 \mathrm{Gbps}$ & $80 \mathrm{Gbps}$ & $100 \mathrm{Gbps}$ & $200 \mathrm{Gbps}$ \\
\hline Sequence Length & 32768 & 32768 & 32768 & 32768 & 32768 & 32768 \\
\hline Samples per bit & 1 & 1 & 1 & 1 & 1 & 1 \\
\hline Number of Samples & 32768 & 32768 & 32768 & 32768 & 32768 & 32768 \\
\hline Sample Rate & $20 \mathrm{Gbps}$ & $40 \mathrm{Gbps}$ & $60 \mathrm{Gbps}$ & $80 \mathrm{Gbps}$ & $100 \mathrm{Gbps}$ & $200 \mathrm{Gbps}$ \\
\hline Symbol rate & $5 \mathrm{Gbps}$ & $10 \mathrm{Gbps}$ & $15 \mathrm{Gbps}$ & $20 \mathrm{Gbps}$ & $25 \mathrm{Gbps}$ & $50 \mathrm{Gbps}$ \\
\hline Sensitivity & $-100 \mathrm{~dB}$ & $-100 \mathrm{~dB}$ & $-100 \mathrm{~dB}$ & $-100 \mathrm{~dB}$ & $-100 \mathrm{~dB}$ & $-100 \mathrm{~dB}$ \\
\hline Resolution & 0.1 & 0.1 & 0.1 & 0.1 & 0.1 & 0.1 \\
\hline \multicolumn{7}{|l|}{ Loop control } \\
\hline \multirow{2}{*}{\multicolumn{4}{|c|}{$\begin{array}{l}\text { Number of Loops } \\
\text { Optical Fiber Parameters }\end{array}$}} & \multicolumn{3}{|l|}{2} \\
\hline \multirow{2}{*}{\multicolumn{4}{|c|}{ Attenuation }} & & & \\
\hline & & & & \multicolumn{3}{|c|}{$0.2 \mathrm{~dB} / \mathrm{Km}$} \\
\hline \multicolumn{4}{|l|}{ Dispersion } & \multicolumn{3}{|c|}{$16.75 \mathrm{ps} / \mathrm{nm}-\mathrm{Km}$} \\
\hline \multicolumn{4}{|l|}{ PMD Coefficient } & \multicolumn{3}{|l|}{0.05} \\
\hline \multicolumn{4}{|l|}{ Effective Area } & \multicolumn{3}{|l|}{$80 \mu \mathrm{m}^{2}$} \\
\hline
\end{tabular}

\section{Result and Discussion}

BER values have been evaluated for different bit-rates and fiber lengths for each modulation format. After that the BER values are arranged in two patternss: the first one is BER versus bit-rate for each fiber length. And the second one is BER versus fiber-length for each bit-rate. Some of the points found by simulation are listed below:

1. At higher bit rate of operation, BER decreases for DP SP QPSK 16 QAM and increases for SP DP QPSK.

2. For SP DP 16 QAM, BER value is almost constant over the length but changes with bit rate. As bit rate increases the BER values decreases.

3. For SP SP 16 QAM, there are little variations in BER over the length as well as over the bit rates

4. For DP SP QPSK 16 QAM, the BER decreases with bit rate increases and almost constant over the length.

5. For SP DP QPSK, BER increases with bit rate and slightly increasing over the length.

\subsection{BER Characteristics:}

The BER characteristics is one of the popular method to analyse the effectiveness of a modulation format. Next four subsections explain the four chosen OFDM formats in terms of BER values over fiber-length and bit-rate.

\subsubsection{SP DP 16 QAM CO-OFDM}

The BER values corresponding to fiber-lengths and bit-rates of layout are listed in table 2. BER versus Bit Rate and versus fiber-length graphs for SP DP 16-QAM CO-OFDM are shown in figure 5 and 6 respectively. The bit error rate decreases over increase in bit-rate (in the range selected for simulation) for all fiber-lengths. The BER values remain almost constant over all fiber-lengths upto $500 \mathrm{Km}$ after which it decreases. This decrease is more rapid for lower bitrates. Approaching $1000 \mathrm{Km}$ length, all bit-rates tend to attain same BER value which is the lowest one.

Table 2. BER values for various bit-rates and fiber lengths

\begin{tabular}{|c|c|c|c|c|c|}
\hline FIBER Length $(\mathrm{Km})$ & \multirow[t]{2}{*}{$\mathbf{0}$} & \multirow[t]{2}{*}{100} & \multirow[t]{2}{*}{300} & \multirow[t]{2}{*}{500} & \multirow[t]{2}{*}{1000} \\
\hline BIT RATE (Gbps) & & & & & \\
\hline 40 & 1.466497 & 1.479552 & 1.46823 & 1.466844 & 0.826267 \\
\hline 60 & 0.866159 & 0.868335 & 0.868063 & 0.873096 & 0.656794 \\
\hline 80 & 0.72041 & 0.712928 & 0.717541 & 0.713378 & 0.596252 \\
\hline 100 & 0.654898 & 0.646582 & 0.653878 & 0.649286 & 0.571499 \\
\hline
\end{tabular}




\begin{tabular}{|l|l|l|l|l|l|}
\hline $\mathbf{2 0 0}$ & 0.546843 & 0.547144 & 0.549166 & 0.54452 & 0.51817 \\
\hline
\end{tabular}



Figure 5. BER vs Bit Rate

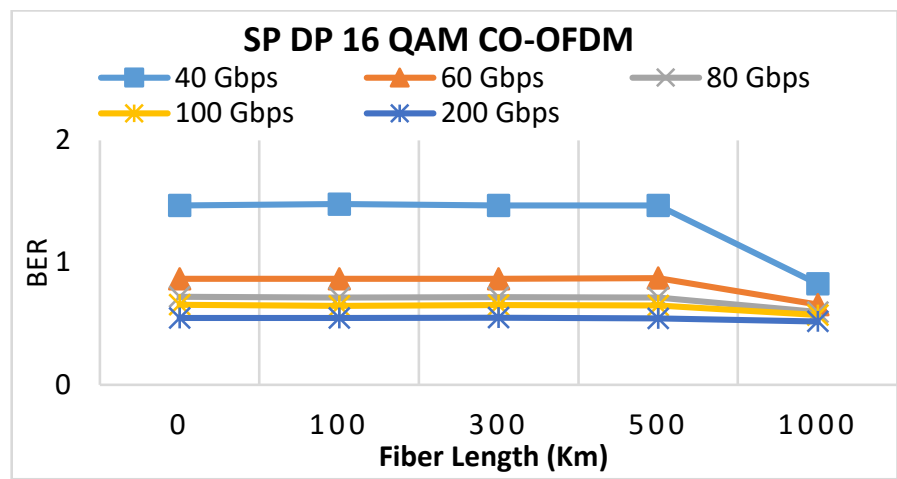

Figure 6. BER vs Fiber Length

\subsubsection{SP SP 16 QAM CO-OFDM}

The BER values corresponding to fiber-lengths and bit-rates of layout are listed in table 3. BER versus Bit Rate and versus fiber-length graphs for SP SP 16-QAM CO-OFDM are shown in figure 7 and 8 respectively. There are very small variations in BER values over bit-rate and over fiber-length. All BER values are same upto two decimal values.

Table 3. BER values for various bit-rates and fiber lengths

\begin{tabular}{|c|c|c|c|c|c|}
\cline { 1 - 5 } \multicolumn{1}{|c|}{ FIBER Length $(\mathbf{K m})$} & $\mathbf{0}$ & $\mathbf{1 0 0}$ & $\mathbf{3 0 0}$ & $\mathbf{5 0 0}$ & $\mathbf{1 0 0 0}$ \\
\cline { 1 - 5 } BIT RATE (Gbps) & & & & & \\
\hline $\mathbf{2 0}$ & 0.438446 & 0.434692 & 0.435181 & 0.439056 & 0.435883 \\
\hline $\mathbf{4 0}$ & 0.435547 & 0.436584 & 0.432831 & 0.43454 & 0.436249 \\
\hline $\mathbf{6 0}$ & 0.431824 & 0.436188 & 0.436554 & 0.434479 & 0.436249 \\
\hline $\mathbf{8 0}$ & 0.431824 & 0.436188 & 0.436554 & 0.434479 & 0.436249 \\
\hline $\mathbf{1 0 0}$ & 0.435547 & 0.437378 & 0.435577 & 0.435638 & 0.435028 \\
\hline $\mathbf{2 0 0}$ & 0.432404 & 0.436829 & 0.43924 & 0.436676 & 0.436615 \\
\hline
\end{tabular}

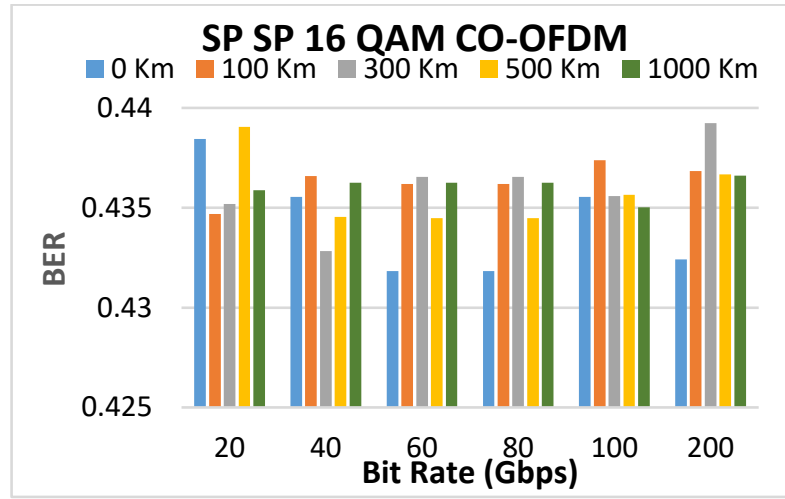

Figure 7. BER vs Bit Rate

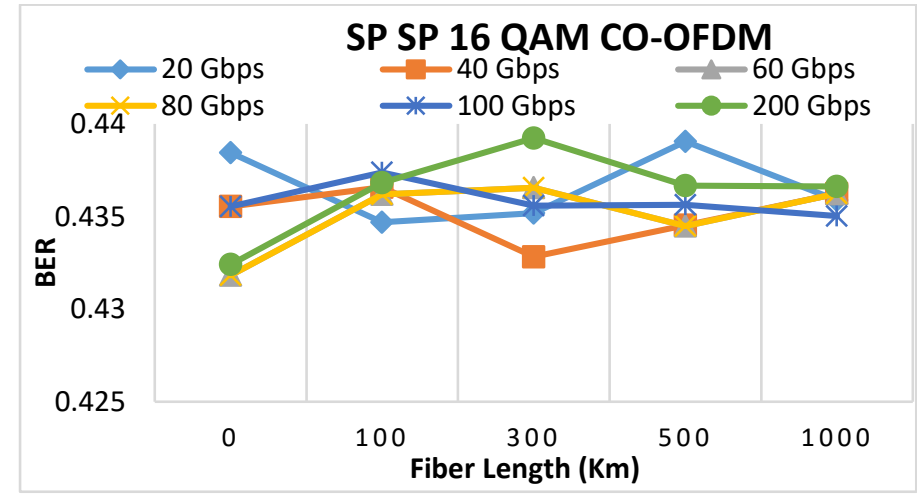

Figure 8. BER vs Fiber Length

\subsubsection{DP SP QPSK 16 QAM CO-OFDM}

The BER values corresponding to fiber-lengths and bit-rates of layout are listed in table 4. BER versus Bit Rate and versus fiber-length graphs for DP SP QPSK 16-QAM CO-OFDM are shown in figure 9 and 10 respectively. Initially with slight increase, the BER values decrease with increase in bit-rate. The BER values remain constant over fiberlength.

Table 4. BER values various bit-rates and fiber lengths

\begin{tabular}{|c|c|c|c|c|c|}
\hline FIBER Length (Km) & 0 & 100 & 300 & 500 & 1000 \\
\cline { 1 - 5 } BIT RATE $(\mathrm{Gbps})$ & & & & & \\
\cline { 1 - 6 } 20 & 1.6647715 & 1.650693 & 1.6457115 & 1.663147 & 1.6515055 \\
\hline
\end{tabular}




\begin{tabular}{|c|c|c|c|c|c|}
\hline 40 & 2.0226105 & 2.0274455 & 2.025455 & 2.021047 & 2.03285 \\
\hline 60 & 1.317258 & 1.328899 & 1.305432 & 1.322062 & 1.298596 \\
\hline 80 & 0.7730575 & 0.770725 & 0.7734915 & 0.777181 & 0.7770725 \\
\hline 100 & 0.6855925 & 0.6803305 & 0.682213 & 0.686607 & 0.6922555 \\
\hline 200 & 0.59063 & 0.5884325 & 0.5867745 & 0.5828775 & 0.5885985 \\
\hline
\end{tabular}

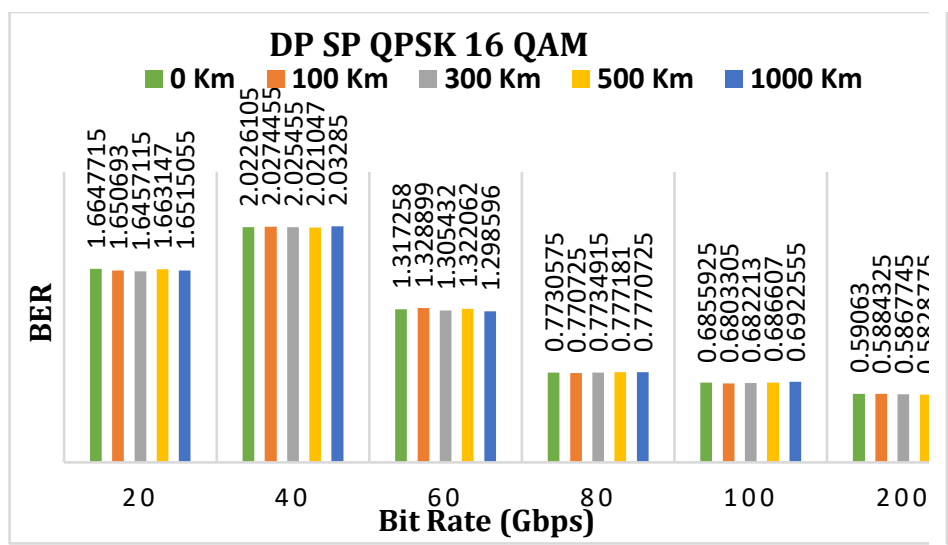

Figure 9. BER vs Bit-Rate

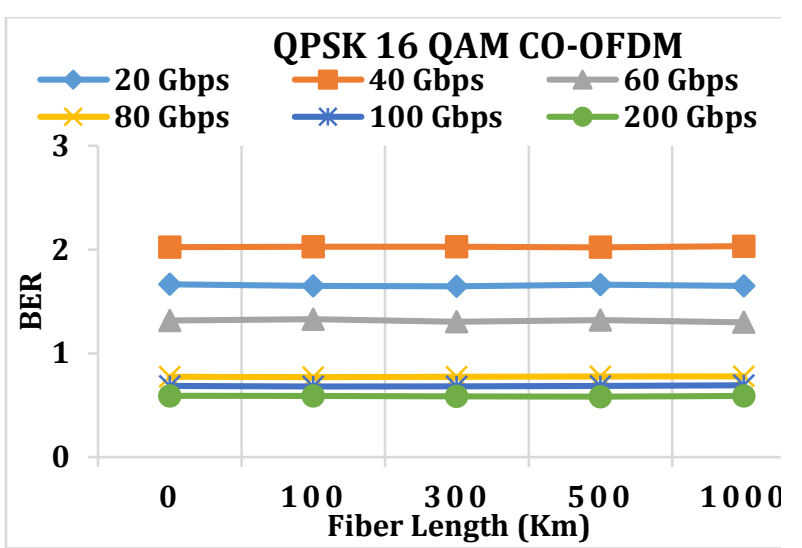

Figure 10. BER vs Fiber Length

\subsubsection{SP DP QPSK CO-OFDM}

The BER values corresponding to fiber-lengths and bit-rates of layout are listed in table 5. BER versus Bit Rate and versus fiber-length graphs for SP DP QPSK CO-OFDM are shown in figure 11 and 12 respectively. BER increases over bit rate. There are sudden increase in BER values from $100 \mathrm{Km}$ to $300 \mathrm{Km}$ length and then become almost constant for all bit-rates except $200 \mathrm{Gbps}$. The $200 \mathrm{Gbps}$ line has little variations over fiber length.

Table 5. BER values for various bit-rates and fiber lengths

\begin{tabular}{|c|c|c|c|c|c|}
\hline FIBER Length (Km) & \multirow[t]{2}{*}{0} & \multirow[t]{2}{*}{100} & \multirow[t]{2}{*}{300} & \multirow[t]{2}{*}{500} & \multirow[t]{2}{*}{1000} \\
\hline BIT RATE (Gbps) & & & & & \\
\hline 20 & 0 & 0 & 0.500483 & 0.497518 & 0.49738 \\
\hline 40 & 0 & 0 & 0.499724 & 0.501862 & 0.503447 \\
\hline 60 & 0.000207 & 0.001862 & 0.498276 & 0.499517 & 0.495863 \\
\hline 80 & 0.008825 & 0.006895 & 0.498001 & 0.500621 & 0.496966 \\
\hline 100 & 0.03013 & 0.0262 & 0.497932 & 0.500758 & 0.500414 \\
\hline 200 & 0.516652 & 0.522117 & 0.528059 & 0.51944 & 0.524648 \\
\hline
\end{tabular}

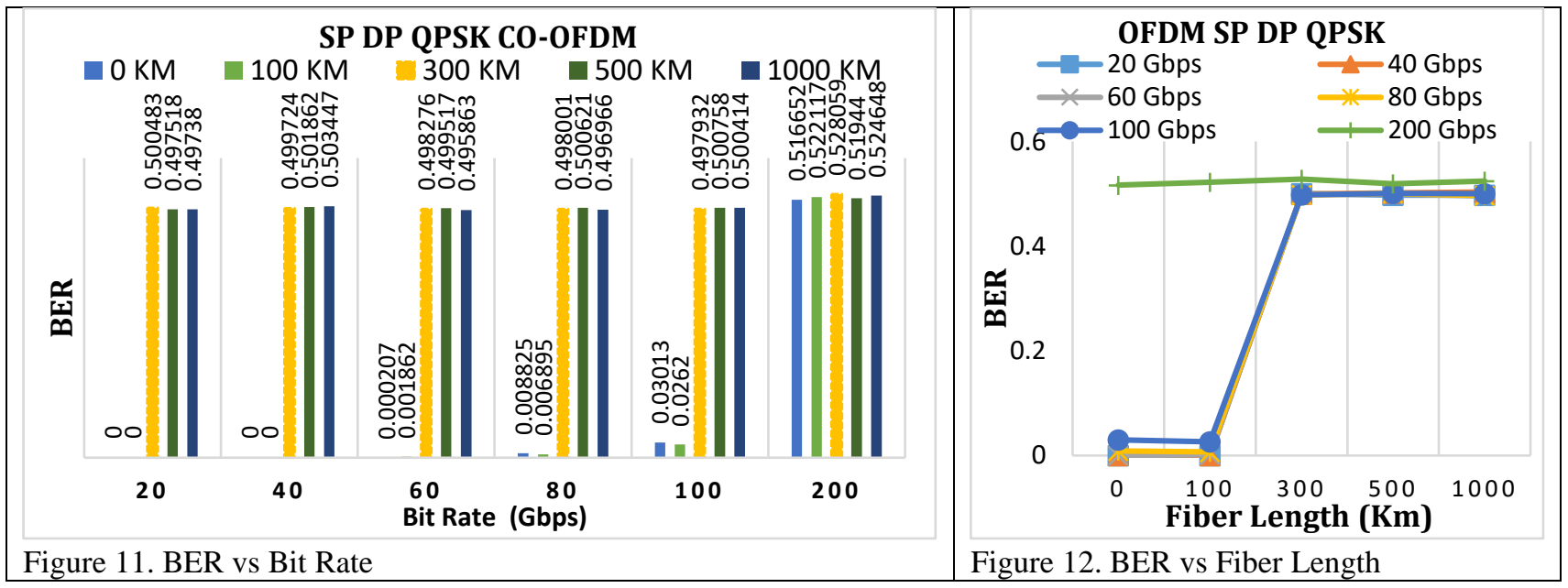




\subsection{Comparative Investigation:}

If $\boldsymbol{w}=$ SP DP 16 QAM CO-OFDM, $\boldsymbol{x}=$ SP SP 16 QAM CO-OFDM, $\boldsymbol{y}=$ DP SP QPSK 16 QAM CO-OFDM and $z=$ SP DP QPSK CO-OFDM and if $\boldsymbol{B} \boldsymbol{w}, \boldsymbol{B} \boldsymbol{x}, \boldsymbol{B} \boldsymbol{y}$ and $\boldsymbol{B} \boldsymbol{z}$ are the corresponding BERs then by $100 \mathrm{Km}$ range the $\boldsymbol{B} \boldsymbol{y}>\boldsymbol{B} \boldsymbol{w}>\boldsymbol{B} \boldsymbol{x}>$ $\boldsymbol{B} z$; for all bit-rates as clear from figure 13 containing various graphs drawn by using table 6 .

Analysis of BER variations as function of bit-rates for various fiber lengths has shown that (i) $\boldsymbol{B} \boldsymbol{y}>\boldsymbol{B} \boldsymbol{w}>\boldsymbol{B} \boldsymbol{x}>\boldsymbol{B} \boldsymbol{z}$ but gap decreases with increase in bit-rates, (ii) all BERs have almost same value at $200 \mathrm{Gbps}$; (iii) the $\boldsymbol{B} \boldsymbol{y}$ becomes almost equal to $\boldsymbol{B} \boldsymbol{x}$ at lengths $300 \mathrm{Km}$ and above as shown in figure 14 containing various graphs drawn using table 7 . Therefore, it is clear that the DP SP QPSK 16 QAM CO-OFDM has the highest value of BER among all formats and the SP DP QPSK CO-OFDM has the lowest value of BER in most of the situations.

Table 6. BER values for a particular bit rate

\begin{tabular}{|c|c|c|c|c|c|}
\hline \multirow{2}{*}{$\begin{array}{c}\text { BIT } \\
\text { RATE } \\
\text { (Gbps) }\end{array}$} & $\begin{array}{l}\text { FIBER } \\
\text { Length }\end{array}$ & \multicolumn{4}{|c|}{ BER } \\
\hline & & $\begin{array}{l}\text { SP DP } \\
16 \text { QAM }\end{array}$ & $\begin{array}{c}\text { SP SP } \\
16 \text { QAM }\end{array}$ & $\begin{array}{c}\text { DP SP } \\
\text { QPSK } \\
16 \text { QAM }\end{array}$ & $\begin{array}{l}\text { SP DP } \\
\text { QPSK }\end{array}$ \\
\hline \multirow[t]{5}{*}{20} & 0 & & 0.438446 & 1.6647715 & 0 \\
\hline & 100 & & 0.434692 & 1.650693 & 0 \\
\hline & 300 & & 0.435181 & 1.6457115 & 0.500483 \\
\hline & 500 & & 0.439056 & 1.663147 & 0.497518 \\
\hline & 1000 & & 0.435883 & 1.6515055 & 0.49738 \\
\hline \multirow[t]{5}{*}{40} & 0 & 1.466497 & 0.435547 & 2.0226105 & 0 \\
\hline & 100 & 1.479552 & 0.436584 & 2.0274455 & 0 \\
\hline & 300 & 1.46823 & 0.432831 & 2.025455 & 0.499724 \\
\hline & 500 & 1.466844 & 0.43454 & 2.021047 & 0.501862 \\
\hline & 1000 & 0.826267 & 0.436249 & 2.03285 & 0.503447 \\
\hline \multirow{5}{*}{60} & 0 & 0.866159 & 0.431824 & 1.317258 & 0.000207 \\
\hline & 100 & 0.868335 & 0.436188 & 1.328899 & 0.001862 \\
\hline & 300 & 0.868063 & 0.436554 & 1.305432 & 0.498276 \\
\hline & 500 & 0.873096 & 0.434479 & 1.322062 & 0.499517 \\
\hline & 1000 & 0.656794 & 0.436249 & 1.298596 & 0.495863 \\
\hline \multirow[t]{5}{*}{80} & 0 & 0.72041 & 0.431824 & 0.7730575 & 0.008825 \\
\hline & 100 & 0.712928 & 0.436188 & 0.770725 & 0.006895 \\
\hline & 300 & 0.717541 & 0.436554 & 0.7734915 & 0.498001 \\
\hline & 500 & 0.713378 & 0.434479 & 0.777181 & 0.500621 \\
\hline & 1000 & 0.596252 & 0.436249 & 0.7770725 & 0.496966 \\
\hline \multirow[t]{5}{*}{100} & 0 & 0.654898 & 0.435547 & 0.6855925 & 0.03013 \\
\hline & 100 & 0.646582 & 0.437378 & 0.6803305 & 0.0262 \\
\hline & 300 & 0.653878 & 0.435577 & 0.682213 & 0.497932 \\
\hline & 500 & 0.649286 & 0.435638 & 0.686607 & 0.500758 \\
\hline & 1000 & 0.571499 & 0.435028 & 0.6922555 & 0.500414 \\
\hline \multirow[t]{5}{*}{200} & 0 & 0.546843 & 0.432404 & 0.59063 & 0.516652 \\
\hline & 100 & 0.547144 & 0.436829 & 0.5884325 & 0.522117 \\
\hline & 300 & 0.549166 & 0.43924 & 0.5867745 & 0.528059 \\
\hline & 500 & 0.54452 & 0.436676 & 0.5828775 & 0.51944 \\
\hline & 1000 & 0.51817 & 0.436615 & 0.5885985 & 0.524648 \\
\hline
\end{tabular}




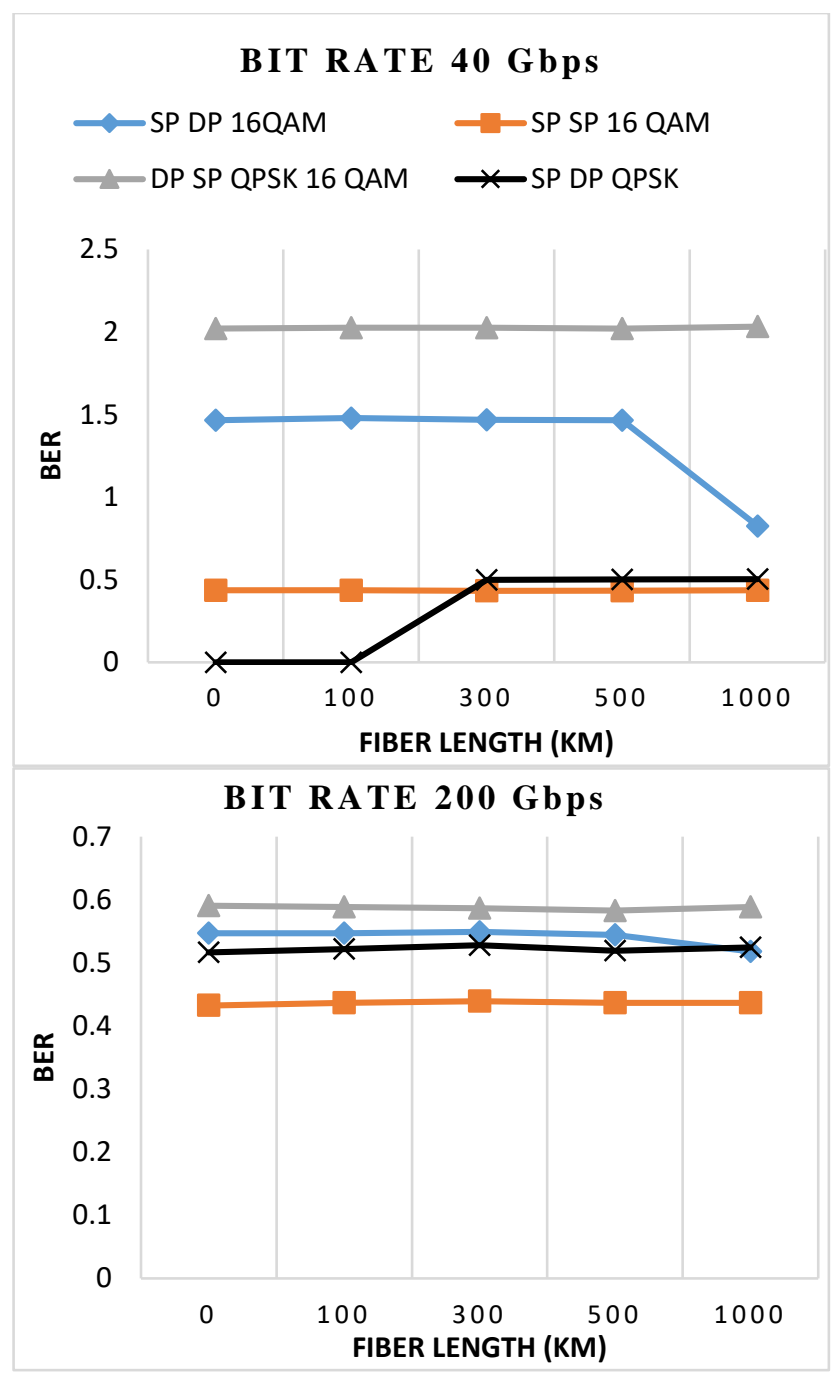

Figure 13. BER characteristics over fiber lengths for different bit rates
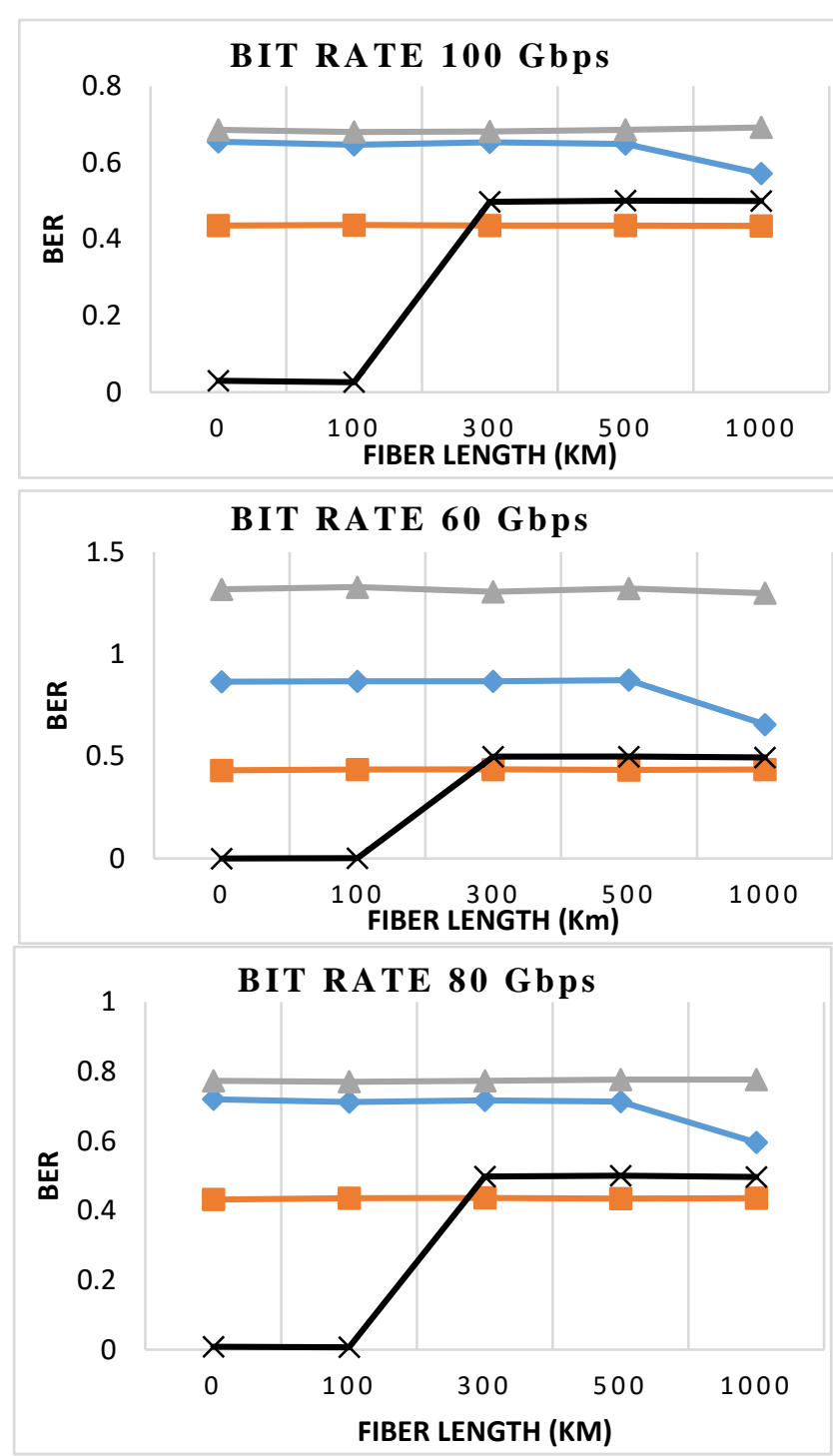

Table 7. BER values for a particular fiber length

\begin{tabular}{|c|c|c|c|c|c|}
\hline $\begin{array}{c}\text { FIBER } \\
\text { Length } \\
(\mathrm{Km})\end{array}$ & $\begin{array}{c}\text { BIT } \\
\text { RATE } \\
(\mathrm{Gbps})\end{array}$ & \multicolumn{5}{|c|}{ BER } \\
\hline & & $\begin{array}{c}\text { OFDM } \\
\text { CD SP DP } \\
\text { 16 QAM }\end{array}$ & $\begin{array}{c}\text { OFDM CD } \\
\text { SP SP } \\
\text { 16 QAM }\end{array}$ & $\begin{array}{c}\text { OFDM CD } \\
\text { DP SP QPSK } \\
\text { 16 QAM }\end{array}$ & $\begin{array}{c}\text { OFDM CD } \\
\text { SP DP } \\
\text { QPSK }\end{array}$ \\
\hline 0 & 20 & & 0.438446 & 1.6647715 & 0 \\
\hline & 40 & 1.466497 & 0.435547 & 2.0226105 & 0 \\
\hline & 60 & 0.866159 & 0.431824 & 1.317258 & 0.000207 \\
\hline & 80 & 0.72041 & 0.431824 & 0.7730575 & 0.008825 \\
\hline & 100 & 0.654898 & 0.435547 & 0.6855925 & 0.03013 \\
\hline & 200 & 0.546843 & 0.432404 & 0.59063 & 0.516652 \\
\hline 100 & 20 & & 0.434692 & 1.650693 & 0 \\
\hline & 40 & 1.479552 & 0.436584 & 2.0274455 & 0 \\
\hline & 60 & 0.868335 & 0.436188 & 1.328899 & 0.001862 \\
\hline & 80 & 0.712928 & 0.436188 & 0.770725 & 0.006895 \\
\hline & 100 & 0.646582 & 0.437378 & 0.6803305 & 0.0262 \\
\hline
\end{tabular}




\begin{tabular}{|c|c|c|c|c|c|}
\hline & 200 & 0.547144 & 0.436829 & 0.5884325 & 0.522117 \\
\hline 300 & 20 & & 0.435181 & 1.6457115 & 0.500483 \\
\hline & 40 & 1.46823 & 0.432831 & 2.025455 & 0.499724 \\
\hline & 60 & 0.868063 & 0.436554 & 1.305432 & 0.498276 \\
\hline & 80 & 0.717541 & 0.436554 & 0.7734915 & 0.498001 \\
\hline & 100 & 0.653878 & 0.435577 & 0.682213 & 0.497932 \\
\hline & 200 & 0.549166 & 0.43924 & 0.5867745 & 0.528059 \\
\hline 500 & 20 & & 0.439056 & 1.663147 & 0.497518 \\
\hline & 40 & 1.466844 & 0.43454 & 2.021047 & 0.501862 \\
\hline & 60 & 0.873096 & 0.434479 & 1.322062 & 0.499517 \\
\hline & 80 & 0.713378 & 0.434479 & 0.777181 & 0.500621 \\
\hline & 100 & 0.649286 & 0.435638 & 0.686607 & 0.500758 \\
\hline & 200 & 0.54452 & 0.436676 & 0.5828775 & 0.51944 \\
\hline 1000 & 20 & & 0.435883 & 1.6515055 & 0.49738 \\
\hline & 40 & 0.826267 & 0.436249 & 2.03285 & 0.503447 \\
\hline & 60 & 0.656794 & 0.436249 & 1.298596 & 0.495863 \\
\hline & 80 & 0.596252 & 0.436249 & 0.7770725 & 0.496966 \\
\hline & 100 & 0.571499 & 0.435028 & 0.6922555 & 0.500414 \\
\hline & 200 & 0.51817 & 0.436615 & 0.5885985 & 0.524648 \\
\hline
\end{tabular}






Figure 14. BER characteristics over bit rates for different fiber lengths

\section{Conclusion}

The comparative analysis has been presented in this paper and four CO-OFDM formats are arranged in decreasing BER values obtained through simulation results. Generally, the DP SP QPSK 16 QAM CO-OFDM has the highest value of BER among all formats and the SP DP QPSK CO-OFDM has the lowest value of BER. At higher bit rate of operation, BER decreases for DP SP QPSK 16 QAM and increases for SP DP QPSK. For SP DP 16 QAM, BER value is almost constant over the length but changes with bit rate. As bit rate increases the BER value decreases. For SP SP 16 QAM, there are little variations in BER over the length as well as over the bit rates. For DP SP QPSK 16 QAM, the BER decreases with bit rate increases and almost constant over the length. For SP DP QPSK, BER increases with bit rate and slightly increasing over the length. If $\boldsymbol{w}=$ SP DP 16 QAM CO-OFDM, $\boldsymbol{x}=$ SP SP 16 QAM CO-OFDM, $\boldsymbol{y}=$ DP SP QPSK 16 QAM CO-OFDM and $z=$ SP DP QPSK CO-OFDM and if $\boldsymbol{B} \boldsymbol{w}, \boldsymbol{B} \boldsymbol{x}, \boldsymbol{B} \boldsymbol{y}$ and $\boldsymbol{B} \boldsymbol{z}$ are the corresponding BERs then by $100 \mathrm{Km}$ range, $\boldsymbol{B} \boldsymbol{y}>\boldsymbol{B} \boldsymbol{w}>\boldsymbol{B} \boldsymbol{x}>\boldsymbol{B} \boldsymbol{z}$ pattern has been observed. It is true for all bit-rates. Analysis of BER variations as function of bit-rates for various fiber lengths has shown that, $\boldsymbol{B} \boldsymbol{y}>\boldsymbol{B} \boldsymbol{w}>\boldsymbol{B} \boldsymbol{x}>\boldsymbol{B} \boldsymbol{z}$ always but gap decreases with increase in bit-rates; all BERs have almost same value at $200 \mathrm{Gbps}$ and the $\boldsymbol{B} \boldsymbol{y}$ becomes almost equal to $\boldsymbol{B} \boldsymbol{x}$ at lengths $300 \mathrm{Km}$ and above. The investigations carried out in this paper present the extent of suitability of a particular CO-OFDM format over the other formats for an application. The selection criteria of a CO-OFDM format for a particular 
application can be further strengthened by involving more parameters such as EVM (Error Vector Magnitude), Q-factor and eye diagrams etc.

\section{References}

[1] William Shieh and Ivan Djordjevic "OFDM for Optical Communications" Book, Academic Press (imprint of Elsevier), ISBN: 978-0-12-374879-9, doi:10.1016/C2009-0-19354-6, 2010.

[2] Jian Dang, Liang Wu and Zaichen Zhang, "OFDM Systems for Optical Communication with Intensity Modulation and Direct Detection" Chapter 5, Intech, http://dx.doi.org/10.5772/intechopen.68199, 2017.

[3] W. Shieh, H. Bao, and Y. Tang, "Coherent optical OFDM: theory and design," Opt. Express, vol. 16, no. 2, pp. 841-859, 2008.

[4] X. Luo, "The application of OFDM in optical fiber communication systems." ICEEMS 2019, IOP Conf. Series: Earth and Environmental Science 332 (2019) 042010, IOP Publishing, doi:10.1088/1755-1315/332/4/042010

[5] J. Armstrong, "OFDM for Optical Communications(Invited Tutorial)," J. Light. Technol., vol. 27, no. 3, pp. 189204, 2009.

[6] A. J. Lowery and L. B. Du, "Optical orthogonal division multiplexing for long haul optical communications: A review of the first five years," Opt. Fiber Technol., vol. 17, no. 5, pp. 421-438, 2011.

[7] V. Nair et al, "Performance analysis of direct detection and coherent detection system for optical OFDM using QAM and DPSK," IOSR J. Eng., vol. 3, no. 7, pp. 24-29, 2013.

[8] N. M. A. Aloff, "Coherent OFDM for Optical Communication systems", Ph.D. thesis, 2014, The Islamic University - Gaza Research \& Graduate Affairs, Faculty of Engineering Master of Electrical Engineering Department, Accessed from: https://library.iugaza.edu.ps > thesis, On: 26 june, 2021.

[9] F. Almasoudi, K. Alatawi, and M. A. Matin, "Study of OFDM Technique on RoF Passive Optical Network," Opt. Photonics J., vol. 03, no. 02, pp. 217-224, 2013.

[10] T. Kurt, A. Yongaçog̃lu, and J. Y. Chouinard, "OFDM and externally modulated multi-mode fibers in radio over fiber systems," IEEE Trans. Wirel. Commun., vol. 5, no. 10, pp. 2669-2674, 2006. 